\title{
Effects of alloying and pressure on magnetic properties of itinerant intermetallic compound $\mathrm{UFe}_{2}$
}

\author{
A. S. Panfilov and I. V. Svechkarev \\ B. Verkin Institute for Low Temperature Physics and Engineering, National Academy of Sciences of Ukraine, \\ 47 Lenin ave., 310164, Kharkov, Ukraine \\ E-mail: panfilov@ilt.kharkov.ua \\ P. Diko, M. Mihalik, and A. Zentko \\ Institute of Experimental Physics, Slovak Academy of Sciences, 04353 Kov̌ice, Slovak Republic
}

Received April 7, 1999

\begin{abstract}
The ferromagnetic state of the itinerant compound $\mathrm{UFe}_{2}$ correlated with a peak in the density of states at the Fermi level is well known to be strongly suppressed by replacing Fe with other $3 d$ elements. To separate the effect of change in filling of the band from that of its deformation under alloying, the magnetic susceptibility of both quasi-binary alloys $\mathrm{U}\left(\mathrm{Fe}_{1-x} \mathrm{Me}_{x}\right)_{2}(\mathrm{Me}=\mathrm{Mn}, \mathrm{Co})$ with a varying number of valence electrons and isoelectronic quasi-ternary alloys $\mathrm{U}\left(\mathrm{Fe}_{1-x} \mathrm{~T}_{x}\right)_{2}, \mathrm{U}\left(\mathrm{Fe}_{0.9-x} \mathrm{Mn}_{0.1} \mathrm{~T}_{x}\right)_{2}$ and $\mathrm{U}\left(\mathrm{Fe}_{0.9-x} \mathrm{Co}_{0.1} \mathrm{~T}_{x}\right)_{2}\left(\mathrm{~T}=\mathrm{Mn}_{0.5} \mathrm{Co}_{0.5}\right)$ was studied in the temperature range $4.2 \mathrm{~K} \leq T \leq 300 \mathrm{~K}$. Both the effects were found to play important roles in suppression of the ferromagnetic state in $\mathrm{UFe}_{2}$-based alloys. In addition, the magnetic susceptibility of $\mathrm{U}\left(\mathrm{Fe}_{1-x} \mathrm{Mn}_{x}\right)_{2}$ and $\mathrm{U}\left(\mathrm{Fe}_{1-x} \mathrm{~T}_{x}\right)_{2}$ alloys and $\mathrm{UCo}_{2}$ compound have been studied under pressure up to $4 \mathrm{kbar}$ at $T=78$ and $293 \mathrm{~K}$. The volume dependence of the exchange enhancement in spin paramagnetism of the $\mathrm{UFe}_{2}$ compound and its alloys has been derived from analysis of the pressure effects in the framework of the Stoner model.
\end{abstract}

PACS: 75.20.En, 75.30.Cr, 71.20.Be

\section{Introduction}

In the series of the cubic C15-type Laves phase compounds $\mathrm{UMe}_{2}(\mathrm{Me}=\mathrm{Mn}, \mathrm{Fe}, \mathrm{Co}, \mathrm{Ni})$ only $\mathrm{UFe}_{2}$ is known to be ferromagnetic with $T_{C}=160 \mathrm{~K}$ (see for example [1]) in which the magnetic moment resides mainly on the Fe sites [2]. The extensive magnetization studies of $\mathrm{UFe}_{2}$ [3] show that the temperature dependence of the spontaneous moment can be fitted well by the sum of spin-wave and Stoner-excitation contributions. The resulted spinwave and Stoner parameters and the critical exponents near the magnetic phase transition as well are close to values reported for pure $3 d$ ferromagnetics. These facts, along with the large electronic specific heat value [4], strongly suggest the itinerant character of magnetism in the $\mathrm{UFe}_{2}$ compound.

To explain tentatively the origin of magnetism in the $\mathrm{UMe}_{2}$ compounds the simple band approach has been suggested [5] which assumes the transfer of $5 f(6 d)$ valence electron of uranium into the $3 d$ states of transition metal. Then the magnetism of
$\mathrm{UNi}_{2}$ is mainly due to the partially occupied states of $5 f$ band, whereas the magnetic moment on $\mathrm{Fe}$ atom in $\mathrm{UFe}_{2}$ is caused by the unfilled $3 d$ band. In this context, a rather weak spin paramagnetism of $\mathrm{UCo}_{2}$ can easily be explained by the minimum in the density of states between $3 d$ and $5 f$ bands. Self-consistent band structure studies of the $\mathrm{UMn}_{2}-$ $\mathrm{UNi}_{2}$ cubic Laves phase systems [6-8] have shown that the situation is more complicated due to a strong hybridization between $3 d$ and $5 f(6 d)$ bands. The calculated density of states (DOS) contains the partial contribution of $3 d$ state which to some extent reminds of a rigid band and becomes progressively filled as we go from $\mathrm{UMn}_{2}$ to $\mathrm{UNi}_{2}$. In addition, there is the uranium $5 f$ band which is also relatively rigid but stays pinned at the Fermi level in such a way that its occupation number is kept almost constant $\left(n_{f} \sim 2.7\right.$ electrons per $\mathrm{U}$ atom $[7,8]$ ). As a consequence, the $5 f$ contribution to the density of states (DOS) at the Fermi level appears to be rather high and roughly constant in magnitude for all mentioned compounds. 
For $\mathrm{UFe}_{2}$, the calculated ground state was found to be ferromagnetic with iron spin moment of $0.7 \mu_{B}$ [7]. This value is in quite good agreement with the experimental one $\left(0.59 \mu_{B}[2]\right)$. However, the spin moment on uranium appears to be $-0.5 \mu_{B}$ and antiparallel to the iron moment, which contradicts the experimentally observed small value $(0.06$ $\mu_{B}$ [2]). The reason for this discrepancy is a substantial cancellation of the $5 f$ spin moment by the orbital contribution which is due to spin-orbit coupling [9-11].

As can be seen from the example of $\mathrm{UFe}_{2}$, the available band structure calculations are in reasonable agreement with experiments, therefore, supporting an itinerant description for both $3 d$ and $5 f$ electrons in the mentioned systems. However, many of the details of quantitative agreement between theory and experimental data have yet to be worked out. Thus, for $\mathrm{UMn}_{2}$ the calculated Stoner criterion for ferromagnetism was found to be just fulfilled $[6,7]$, that is in rather poor agreement with the experiments in which the only moderately enhanced paramagnetic state and no evidence for magnetic ordering have been observed [12, 13]. In addition, the inherent fine structure of calculated DOS at the Fermi level along with large enhancement factor favors the strong temperature dependence of the magnetic susceptibility (as in the case of strongly enhanced paramagnetics such as $\mathrm{Pd}$, $\mathrm{TiBe}_{2}, \mathrm{Ni}_{3} \mathrm{Ga}$, and so on). In fact, this dependence for $\mathrm{UMn}_{2}$ was found to be weak [12, 14].

The experimental studies of the magnetic properties of pseudobinary $\mathrm{U}\left(\mathrm{Fe}_{1-x} \mathrm{Me}_{x}\right)_{2}$ alloys show that the ferromagnetic state of $\mathrm{UFe}_{2}$ is strongly suppressed by substitution of iron with other $3 d$ elements [1]. In order to estimate a critical concentration for disappearance of the ferromagnetic state in $\mathrm{U}\left(\mathrm{Fe}_{1-x} \mathrm{Me}_{x}\right)_{2}$ alloys the calculation were made [8] using simulation of the electronic structure of selected disordered alloys by that of ordered stoichiometric compounds with the same number of valence electrons, namely $\mathrm{U}_{2} \mathrm{Fe}_{3} \mathrm{Mn}$ and $\mathrm{UFeMn}$. Based on a quite reasonable agreement of calculation with experimental data, the authors of [8] concluded that influence of a disorder in the $3 d$ metal sublattice is of relatively minor importance. In addition, for these systems the validity of the rigid-band was found to be good which is favoured by a very similar shape and extension of the $3 d$ wave function of iron and manganese in these types of pseudobinary alloys. On the other hand, the experimental data for $\mathrm{U}\left(\mathrm{Mn}_{1-x} \mathrm{Co}_{x}\right)_{2}$ alloys [14] exhibit a well-defined maximum of the magnetic susceptibility at $x=0.5$ that corresponds to the same number of valence electrons as for $\mathrm{UFe}_{2}$ compound. However, some difference in the magnetic properties of $\mathrm{U}\left(\mathrm{Mn}_{0.5} \mathrm{Co}_{0.5}\right)_{2}$ alloy and $\mathrm{UFe}_{2}$ reveals that the simple rigid-band model fails to some extent to describe these properties.

The high pressure experimental $[4,15]$ and theoretical $[7,9]$ studies of the magnetic properties of $\mathrm{UFe}_{2}$ show that its ferromagnetic state is strongly pressure dependent. Thus, the calculated spin magnetism disappears for a pressure between 400 and $500 \mathrm{kbar}$. With a linear extrapolation of the experimental value of pressure derivative for the Curie temperature, $d T_{C} / d P=-0.52 \mathrm{~K} / \mathrm{kbar}$ [4], the critical pressure appears to be of about $300 \mathrm{kbar}$ that is somewhat below the theoretical estimate. There is the same degree of agreement between the calculated pressure dependence of the magnetic moment, $d \ln m / d P=-3.0$ Mbar [9], and the experimental one, $d \ln m / d P=-4.8$ Mbar [4]. In addition, the rhombohedrical distortion of $\mathrm{UFe}_{2}$ is reported to occur just below the Curie temperature [1] that supports a large magnetoelastic effects in this compound.

As is evident from the foregoing, the electronic structure and magnetic properties of $\mathrm{UFe}_{2}$ and related compounds are rather complicated and in order to gain a better understanding these properties further theoretical and experimental studies are required.

In present work we give results of the magnetic susceptibility measurements for quasi-ternary $\mathrm{U}\left(\mathrm{Fe}_{1-x} \mathrm{~T}_{x}\right)_{2}, \quad \mathrm{U}\left(\mathrm{Fe}_{0.9-x} \mathrm{Mn}_{0.1} \mathrm{~T}_{x}\right)_{2}$ and $\mathrm{U}\left(\mathrm{Fe}_{0.9-x} \mathrm{Co}_{0.1} \mathrm{~T}_{x}\right)_{2} \quad\left(\mathrm{~T}=\mathrm{Mn}_{0.5} \mathrm{Co}_{0.5}\right)$ alloys and quasi-binary $\mathrm{U}\left(\mathrm{Fe}_{1-x} \mathrm{Me}_{x}\right)_{2}(\mathrm{Me}=\mathrm{Mn}, \mathrm{Co})$ alloys in the temperature range $4.2 \mathrm{~K} \leq T \leq 300 \mathrm{~K}$. The experimental data on the concentration dependence of the magnetic properties for both isoelectronic quasi-ternary alloys and quasi-binary alloys with a varying number of valence electrons were used to separate the effect of the change in occupation of the band from that of its deformation under alloying. To estimate a role of the chemical pressure caused by the change in a lattice parameter, the pressure effects on the magnetic susceptibility of $\mathrm{U}\left(\mathrm{Fe}_{1-x} \mathrm{Mn}_{x}\right)_{2}$ and $\mathrm{U}\left(\mathrm{Fe}_{1-x} \mathrm{~T}_{x}\right)_{2}$ alloys and $\mathrm{UCo}_{2}$ compound have been studied at $T=78$ and $293 \mathrm{~K}$ and pressure up to $4 \mathrm{kbar}$. From analysis of the experimental data in the framework of the Stoner model, volume dependence of the exchange enhancement in spin paramagnetism of the $\mathrm{UFe}_{2}$ compound and its alloys has been derived. 


\section{Experimental technique and results}

The polycrystalline samples were prepared by melting high purity metals in an induction furnace under an argon atmosphere. The cubic Laves phase structure was confirmed by $\mathrm{x}$-ray study. The composition of the samples was determined by chemical analysis.

A Faraday microbalance was used for susceptibility measurements in the temperature range $4.2-300 \mathrm{~K}$ in magnetic fields up to $10 \mathrm{kG}$. To eliminate small ferromagnetic contributions, which are probably due to the presence of some phases with higher $T_{C}$ or unreacted transition metals, the corrected values of the magnetic susceptibility $\chi$ were determined by extrapolation of the measured $\chi\left(H^{-1}\right)$ dependence to $H^{-1}=0$. The examples of the temperature dependence of reciprocal susceptibility for quasi-ternary alloys $\mathrm{U}\left(\mathrm{Fe}_{1-x} \mathrm{~T}_{x}\right)_{2}$ $\left(\mathrm{T}=\mathrm{Mn}_{0.5} \mathrm{Co}_{0.5}\right)$ are shown in Fig. 1. It can be seen that for $T \geq 50 \mathrm{~K}$ the $\chi(T)$ values obey the modified Curie-Weiss law

$$
\chi(T)=\chi_{0}+C /(T-\Theta),
$$

where $\chi_{0}$ is temperature independent contribution (at $T<50 \mathrm{~K}$ for some alloys the $1 / T$ contribution, which is presumably of parasitic superparamagnetism nature, manifests itself). In Fig. 2 we plotted the Curie-Weiss parameters vs. concentration,

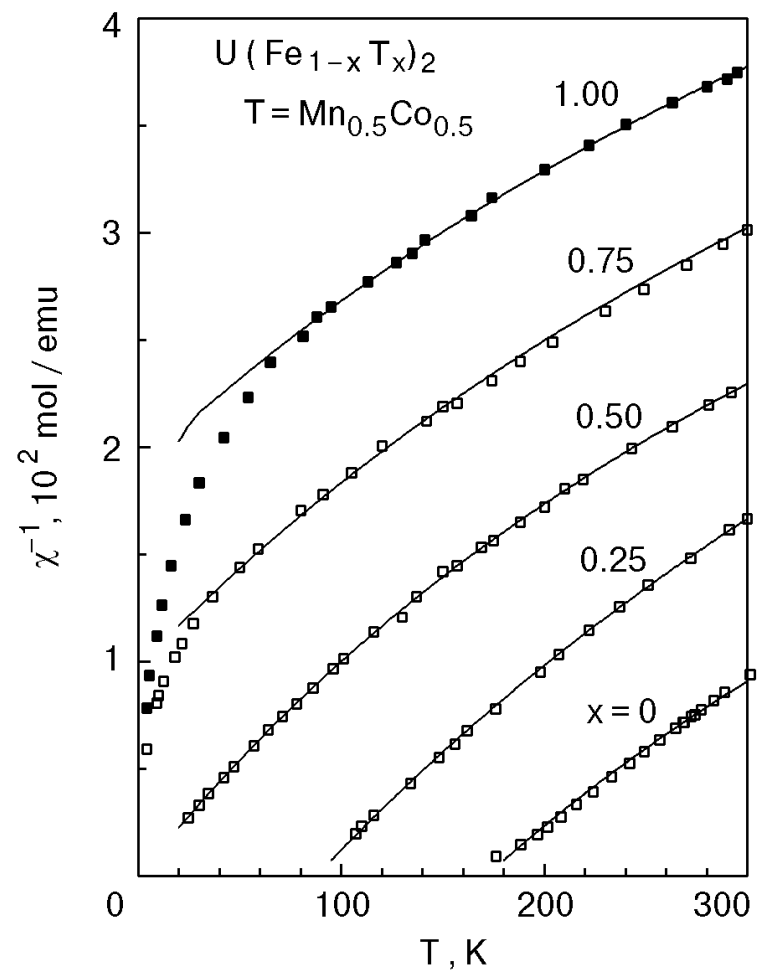

Fig. 1. Temperature dependences of the magnetic susceptibility for ternary isoelectronic alloys $\mathrm{U}\left(\mathrm{Fe}_{1-x} \mathrm{~T}_{x}\right)_{2} \quad\left(\mathrm{~T}=\mathrm{Mn}_{0.5} \mathrm{Co}_{0.5}\right)$. Full lines correspond to fitting by Eq. (1). obtained from experimental data at $T \geq 50 \mathrm{~K}$ for $\mathrm{U}\left(\mathrm{Fe}_{1-x} \mathrm{~T}_{x}\right)_{2}, \quad \mathrm{U}\left(\mathrm{Fe}_{0.9-x} \mathrm{Mn}_{0.1} \mathrm{~T}_{x}\right)_{2} \quad$ and $\mathrm{U}\left(\mathrm{Fe}_{0.9-x} \mathrm{Co}_{0.1} \mathrm{~T}_{x}\right)_{2}$ alloys. It is evident from Fig. 2 that only the Curie temperature $\Theta$ is strongly affected by alloying whereas the Curie constant $C$ and contribution $\chi_{0}$ vary only slightly. It should be noted that the observed essential suppression of the ferromagnetic state in isoelectronic alloys cannot be explained by the simple rigid-band model.

For binary alloys studied, $\mathrm{U}\left(\mathrm{Fe}_{1-x} \mathrm{Mn}_{x}\right)_{2}$ $(x=0.1,0.25,0.5,1.0)$ and $\mathrm{U}\left(\mathrm{Fe}_{1-x} \mathrm{Co}_{x}\right)_{2}(x=0.1$, $0.2)$, the $\chi(T)$ dependences are similar to those of Refs. 12, 14, 16 and Refs. 17, 18, respectively, and are not shown here.

The study of the magnetic susceptibility under uniform pressures up to $4 \mathrm{kbar}$ at liquid nitrogen and room temperatures was carried out by two methods: the Faraday method with the pendulum magnetometer placed directly into the high-pressure chamber [19], and the levitation method [20]. The relative error did not exceed $0.05 \%$ in either case. As an example, the $\chi(P)$ dependence for $\mathrm{UFe}_{2}$ at different temperatures is given in Fig. 3. It shows the magnitude of the pressure effect and its linear behavior, yielding values for $d \ln \chi / d P$. The $d \ln \chi / d P$ data for $\mathrm{U}\left(\mathrm{Fe}_{1-x} \mathrm{Mn}_{x}\right)_{2}$ and $\mathrm{U}\left(\mathrm{Fe}_{1-x} \mathrm{~T}_{x}\right)_{2}$

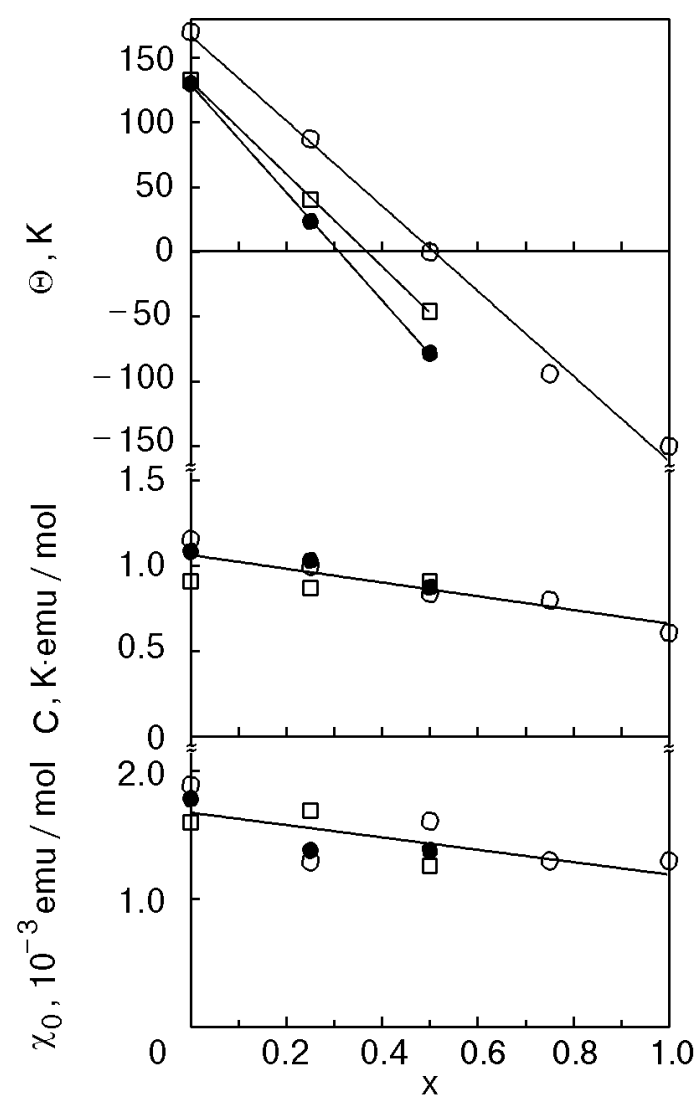

Fig. 2. Concentration dependences of the Curie-Weiss parameters for ternary $\mathrm{UFe}_{2}$-based alloys: $\mathrm{U}\left(\mathrm{Fe}_{1-x} \mathrm{~T}_{x}\right)_{2}\left(\mathrm{~T}=\mathrm{Mn}_{0.5} \mathrm{Co}_{0.5}\right)$ (O); $\mathrm{U}\left(\mathrm{Fe}_{0.9-x} \mathrm{Mn}_{0.1} \mathrm{~T}_{\mathrm{x}}\right)_{2}(\bullet) ; \mathrm{U}\left(\mathrm{Fe}_{0.9-x} \mathrm{Co}_{0.1} \mathrm{~T}_{x}\right)_{2}(\square)$. 
( $\left.\mathrm{T}=\mathrm{Mn}_{0.5} \mathrm{Co}_{0.5}\right)$ alloys and $\mathrm{UCo}_{2}$ compound are summarized in Table. Preliminary report on part of the results obtained was given in [21].

Table

Experimental values of the pressure derivative $d \ln \chi / d P$ and the magnetic susceptibility $\chi$ itself at different temperatures for $\mathrm{U}\left(\mathrm{Fe}_{1-x} \mathrm{Me}_{x}\right)_{2}$ alloys $\left(\mathrm{Me}=\mathrm{Mn}\right.$, $\mathrm{Co}$ and $\left.\mathrm{Mn}_{0.5} \mathrm{Co}_{0.5}\right)$

\begin{tabular}{|c|c|c|c|}
\hline Compound & $\begin{array}{c}-d \ln \chi / d P \\
\operatorname{Mbar}^{-1}\end{array}$ & $\begin{array}{c}\chi, \\
10^{-3} \mathrm{emu} / \mathrm{mol}\end{array}$ & $T, \mathrm{~K}$ \\
\hline $\begin{array}{c}\mathrm{U}\left(\mathrm{Fe}_{1-x} \mathrm{Mn}_{x}\right)_{2} \\
x=0.1\end{array}$ & $8.3 \pm 0.5$ & 8.32 & 293 \\
\hline 0.25 & $7.5 \pm 0.5$ & 5.97 & " \\
\hline 0.3 & $7.1 \pm 0.4$ & 5.45 & " \\
\hline 0.5 & $5.2 \pm 0.4$ & 4.05 & " \\
\hline 0.75 & $4.0 \pm 0.3$ & 2.96 & " \\
\hline 1.0 & $3.6 \pm 0.3$ & 2.38 & " \\
\hline 0.25 & $26.0 \pm 1.0$ & 37.9 & 78 \\
\hline 0.3 & $17.2 \pm 0.5$ & 20.8 & " \\
\hline 0.5 & $7.1 \pm 0.5$ & 6.8 & " \\
\hline $\mathrm{U}\left[\mathrm{Fe}_{1-x}\left(\mathrm{Mn}_{0.5} \mathrm{Co}_{0.5}\right)_{x}\right]_{2}$ & & & \\
\hline$x=0.25$ & $6.7 \pm 0.5$ & 6.16 & 293 \\
\hline 0.5 & $6.2 \pm 0.4$ & 4.53 & " \\
\hline 0.75 & $4.9 \pm 0.4$ & 3.48 & " \\
\hline 1.0 & $4.6 \pm 0.3$ & 2.74 & " \\
\hline \multirow[t]{3}{*}{$\mathrm{UFe}_{2}$} & $10.1 \pm 0.5$ & 10.7 & 300 \\
\hline & $10.5 \pm 0.5$ & 11.3 & 293 \\
\hline & $21.2 \pm 1.0$ & 33.1 & 210 \\
\hline \multirow[t]{2}{*}{$\mathrm{UCO}_{2}$} & $2.25 \pm 0.3$ & 1.11 & 300 \\
\hline & $2.1 \pm 0.3$ & 1.18 & 78 \\
\hline
\end{tabular}

\section{Discussion}

In the Stoner theory of itinerant electron magnetism, electron-electron interactions manifest themselves through the enhancement of the Pauli spin susceptibility $\chi_{P}$ by the Stoner factor $S$ :

$$
\chi=S \chi_{P}=\frac{\chi_{P}}{1-\alpha \chi_{P}},
$$

where $\chi_{P}=2 \mu_{B}^{2} N\left(E_{F}\right), N\left(E_{F}\right)$ is density of states at the Fermi level; the molecular field constant $\alpha=\left(1 / 2 \mu_{B}^{2}\right) I, I$ is a parameter of the exchange-correlation interaction between the conduction electrons. For finite temperature, $\chi_{P}(T)$ is determined by the effective density of states at the Fermi level, $N(\mu, T) \equiv N$, given by

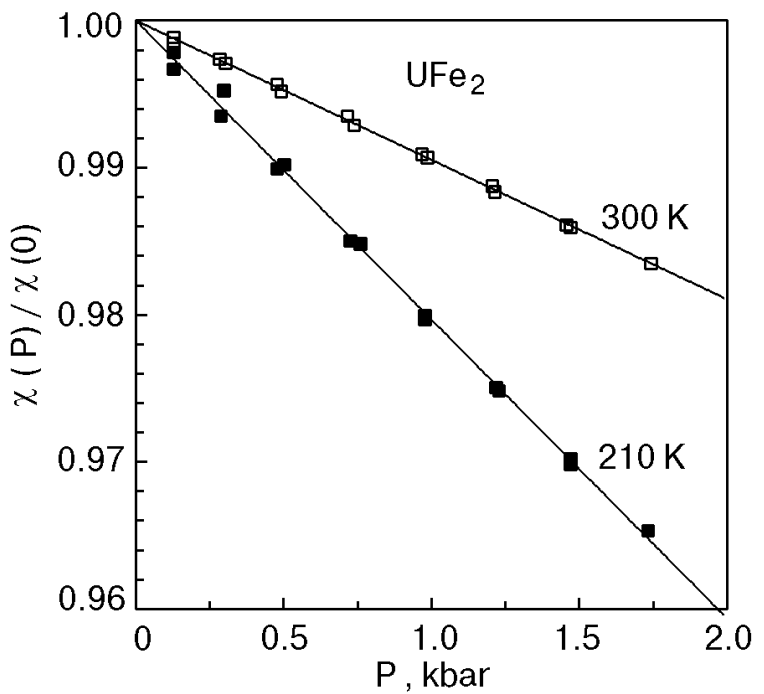

Fig. 3. Pressure dependence of the magnetic susceptibility for $\mathrm{UFe}_{2}$ at two different temperatures normalized to its value at $P=0$.

$$
N(\mu, T)=\int_{0}^{\infty} N(E)[-\partial f(E, \mu, T) / \partial E] d E .
$$

Here $f(E, \mu, T)$ is the Fermi-Dirac distribution function, wherein the chemical potential $\mu(T)$ is determined by assuming the number of band electrons in the system to be constant. In the case $I N \simeq 1$ (for strong itinerant paramagnets or weak ferromagnets at $T \geq T_{C}$ ) the enhanced spin paramagnetism is a dominant contribution to the magnetic susceptibility. Equation (2) will be considered to be appropriate for $\mathrm{UFe}_{2}$ and its alloys studied to analyze the changes of their magnetic properties on alloying and under pressure.

\subsection{Effects of alloying}

In order to discuss the concentration dependence of the magnetic susceptibility, Eq. (2) is conveniently represented by

$$
1 / \chi(T)=1 / \chi_{P}(T)-\alpha .
$$

Then the experimental values of $1 / \chi$ for $\mathrm{U}\left(\mathrm{Fe}_{1-x} \mathrm{~T}_{x}\right)_{2}$ (see Fig. 1) plotted against concentration $x$ appear to be an almost linear function of $x$ with $\partial(1 / \chi) / \partial x$ being roughly temperature independent. From Eq. (4) it follows that

$$
\partial(1 / \chi) / \partial x=\partial\left(1 / \chi_{P}\right) / \partial x-\partial \alpha / \partial x
$$

and the effect of alloying is evidently due to changes in both the density of states at the Fermi level and the molecular field constant $\alpha$. There are 
no strong grounds to believe that $\alpha$ has a detectable concentration dependence. Its magnitude resulted from calculated values of $I(9.3,9.5$ and $11.3 \mathrm{mRy} \cdot c e l l$ for $\mathrm{UMn}_{2}, \mathrm{UFe}_{2}$ and $\mathrm{UCo}_{2}$, respectively $\left.[9,22]\right)$ shows only a weak growing as we go from $\mathrm{UMn}_{2}$ to $\mathrm{UCo}_{2}$. Thus, as a preliminary, we can neglect this effect by assuming $\alpha$ to be constant. It should also be noted that the $\alpha$ value averaged over composition is unchanged with substitution of $\mathrm{Mn}_{0.5} \mathrm{Co}_{0.5}$ for $\mathrm{Fe}$ atoms in $\mathrm{UFe}_{2}$ and related alloys provided that we admit $\alpha$ to be changing linearly along the $\mathrm{UMn}_{2}-$ $\mathrm{UCO}_{2}$ series. Hence, the change in the magnetic properties of the isoelectronic alloys is assumed to be mainly caused by a change in the density of states $N$ under alloying.

One mechanism of this change is the electron scattering on the disorder in the $3 d$ sublattice due to the random occupation by impurity of the $B$ sites in the $A B_{2}$ Laves system. The simplified description of the scattering effect can be given in terms of the effective temperature $T^{*}[23,24]$

$$
T^{*}=\beta x,
$$

where in general case, the coefficient $\beta$ depends on the type of doping impurity. This assumption is demonstrated in Fig. 1 where the most pronounced effect of alloying can be imagined as the temperature shift in $1 / \chi(T)$ dependences according to

$$
1 / \chi(T) \simeq(1 / C)\left(T+T^{*}-\Theta\right) .
$$

Then the coefficient $\beta$ in Eq. (6) can be estimated from the experimental data at any particular temperature as the ratio

$$
\beta=\frac{\partial(1 / \chi) / \partial x}{\partial(1 / \chi) / \partial T}
$$

With the data at room temperature we obtain $\beta=4.3 \pm 0.5 \mathrm{~K}$ per at. $\%$ of $\mathrm{Mn}_{0.5} \mathrm{Co}_{0.5}$ which is the average of the results for $\mathrm{U}\left(\mathrm{Fe}_{1-x} \mathrm{~T}_{x}\right)_{2}$, $\mathrm{U}\left(\mathrm{Fe}_{0.9-x} \mathrm{Mn}_{0.1} \mathrm{~T}_{x}\right)_{2}$ and $\mathrm{U}\left(\mathrm{Fe}_{0.9-x} \mathrm{Co}_{0.1} \mathrm{~T}_{x}\right)_{2}$ alloys. The similar estimate follows directly from the concentration dependence of the paramagnetic Curie temperature (Fig. 2), namely, $\beta=3.7 \pm 0.5 \mathrm{~K}$ per at. $\%$ of $\mathrm{Mn}_{0.5} \mathrm{Co}_{0.5}$. Thus the value of $\beta$ may be considered to be temperature independent and equal to

$$
\beta=4.0 \pm 0.5 \mathrm{~K} / \text { at. } \% \mathrm{Mn} \text { or } \mathrm{Co}
$$

assuming a similarity in the scattering properties of $\mathrm{Mn}$ and Co dopants in $\mathrm{UFe}_{2}$ and related alloys.

As evident from the estimate obtained, the scattering effect plays an important role in suppression of the ferromagnetic state in $\mathrm{UFe}_{2}$ based alloys.
Thus, for the experimental value of the critical concentration $x \simeq 0.3$ for disappearance of ferromagnetism in $\mathrm{U}\left(\mathrm{Fe}_{1-x} \mathrm{Mn}_{x}\right)_{2}$ and $\mathrm{U}\left(\mathrm{Fe}_{1-x} \mathrm{Co}_{x}\right)_{2}$ alloys [1], the reduction in the Curie temperature $T_{C}=160 \mathrm{~K}$ resulted only from the scattering is estimated to be close to $120 \mathrm{~K}$. Therefore the rigid band model for $\mathrm{UFe}_{2}$-based alloys has to be modified to account for this effect.

With the simplified description of scattering effect in terms of the effective temperature, the magnetic susceptibility as a function of the electron number can be derived from the experimental data on $\chi(T, x)$ in $\mathrm{U}\left(\mathrm{Fe}_{1-x} \mathrm{Mn}_{x}\right)_{2}$ and $\mathrm{U}\left(\mathrm{Fe}_{1-x} \mathrm{Co}_{x}\right)_{2}$ alloys by introducing a corresponding substitution of $\left(T-T^{*}\right)$ for argument $T$ in the $\chi(T)$ dependence where for concentrated alloys $T^{*}=\beta x(1-x)$ is used instead of (6).

In Fig. 4 is shown the resulting dependence $\chi$ vs. number of valence electrons per $3 d$ atom, $n$, measured from that for $\mathrm{UFe}_{2}$ at $T=250 \mathrm{~K}$ which points to the essential effect of scattering in the magnetic susceptibility of the alloys studied.

Notice that the temperature and concentration independent contribution $\chi_{0}$ in Eq. (1) becomes the dominant part of the magnetic susceptibility outside the range of $-0.7 \leq n \leq 0.6$. Its value in $\mathrm{UFe}_{2}$ and $\mathrm{UFe}_{2}$-based alloys (see Fig. 2) is close to the magnetic susceptibility of $\mathrm{UCo}_{2}$ at $T=4.2 \mathrm{~K}$ which equals $1.35 \cdot 10^{-3} \mathrm{emu} / \mathrm{mol}$ [4]. A comparison of this value with the estimate of the exchange-enhanced

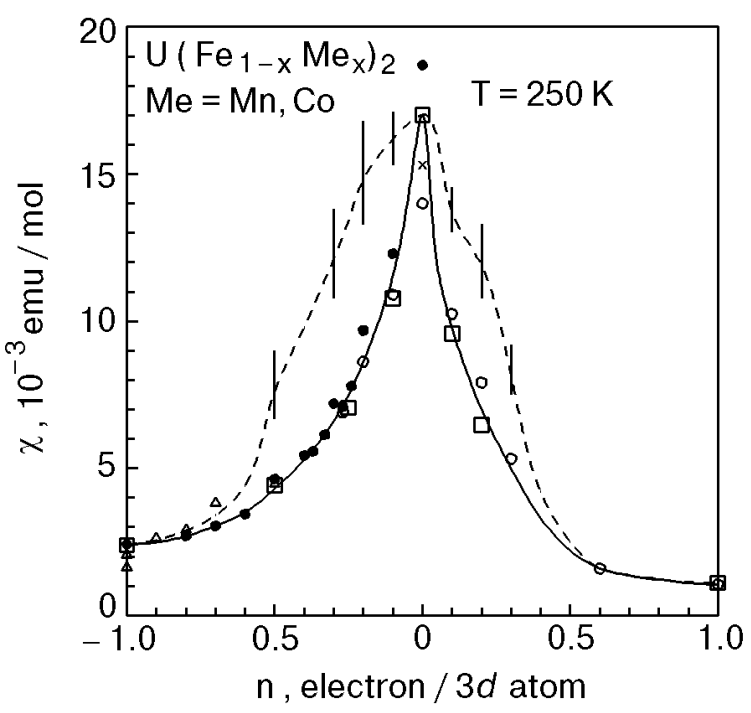

Fig. 4. Magnetic susceptibility of binary alloys $\mathrm{U}\left(\mathrm{Fe}_{1-x} \mathrm{Me}_{x}\right)_{2}$ $(\mathrm{Me}=\mathrm{Mn}, \mathrm{Co})$ at $T=250 \mathrm{~K}$ as a function of the electron number measured from that of $\mathrm{UFe}_{2}$ (full line): our data ( $\square$ ); Refs. $3(x) ; 12(\bullet) ; 14(\Delta) ; 17,18(\bigcirc)$. The dashed curve is the same dependence obtained by taking into account the scattering effect in terms of the effective temperature. Bars on the curve correspond to errors in estimation of the magnitude of $\beta$ (see Eq. (9)). 


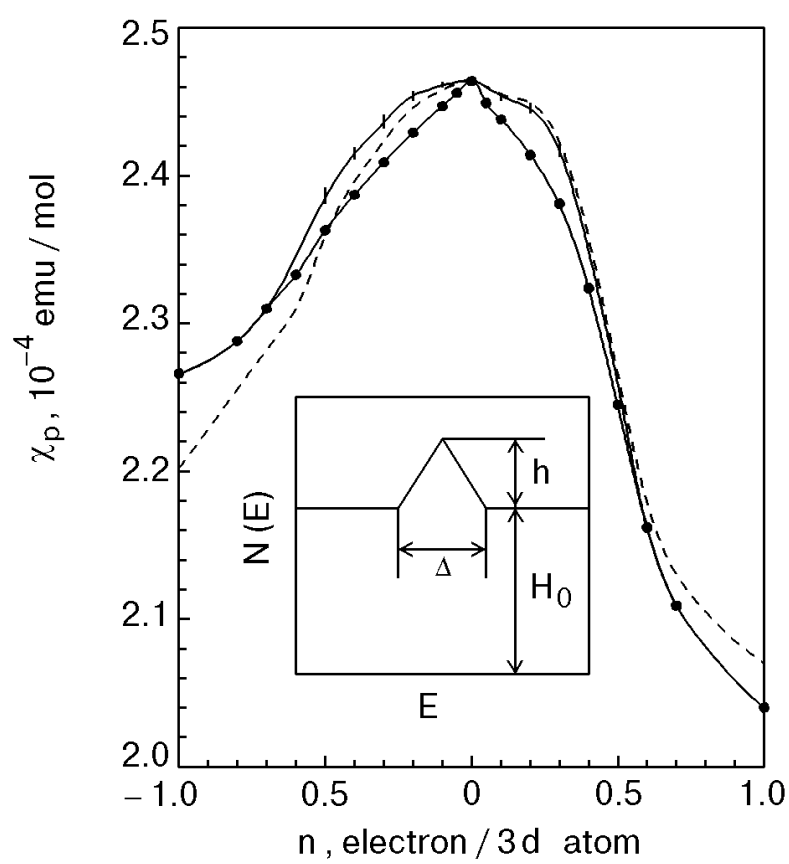

Fig. 5. The Pauli susceptibility at $T=250 \mathrm{~K}$ as a function of the electron number measured from that of $\mathrm{UFe}_{2}$ : the curve marked by (-) corresponds to the experimental data in binary alloys, the solid line includes corrections for the scattering and the dashed line is the same for the constant volume conditions. Inset: a sketch of the model density of state curve.

spin susceptibility in $\mathrm{UCo}_{2}, 1.28 \cdot 10^{-3} \mathrm{emu} / \mathrm{mol}$ [9], shows that it is reasonable to suggest the contribution $\chi_{0}$ to be also of the spin nature. Therefore, for all alloys under consideration the magnetic susceptibility as a whole may be treated as being enhanced spin paramagnetism, that proves the use of the susceptibility data to recover the DOS curve for $\mathrm{UFe}_{2}$ through the estimation of the Pauli spin susceptibility, $\chi_{P}(T) \propto N\left(E_{F}, T\right)$, in framework of Eq. (2).

Figure 5 shows the $\chi_{P}(n)$ dependence for the range $-1.0 \leq n \leq 1.0$ and $T=250 \mathrm{~K}$ which has been obtained from the $\chi(n)$ data of Fig. 4 by using Eq. (2) with calculated for $\mathrm{UFe}_{2}$ value $\alpha=4.0 \cdot 10^{3} \mathrm{~mol} / \mathrm{emu}(I=0.0095 \mathrm{Ry}[9])$. As seen, this dependence, and hence the dependence $N(n)$, takes the form of a peak and its maximum corresponds to the $\mathrm{UFe}_{2}$ compound. In addition, some asymmetry in $\chi_{P}(n)$ about $n=0$ decreases noticeably after applying the corrections for the volume changes on alloying obtained with the experimental data for pressure dependence of the magnetic susceptibility (see Table 1 and discussion of the magnetovolume effects) and for the lattice parameters in alloys $[17,18]$. As is clear from Fig. 5, the corrections of $\chi_{P}(n)$ for both scattering and volume change in alloys are rather small in themselves. Nevertheless, their effects on the magnetic proper-

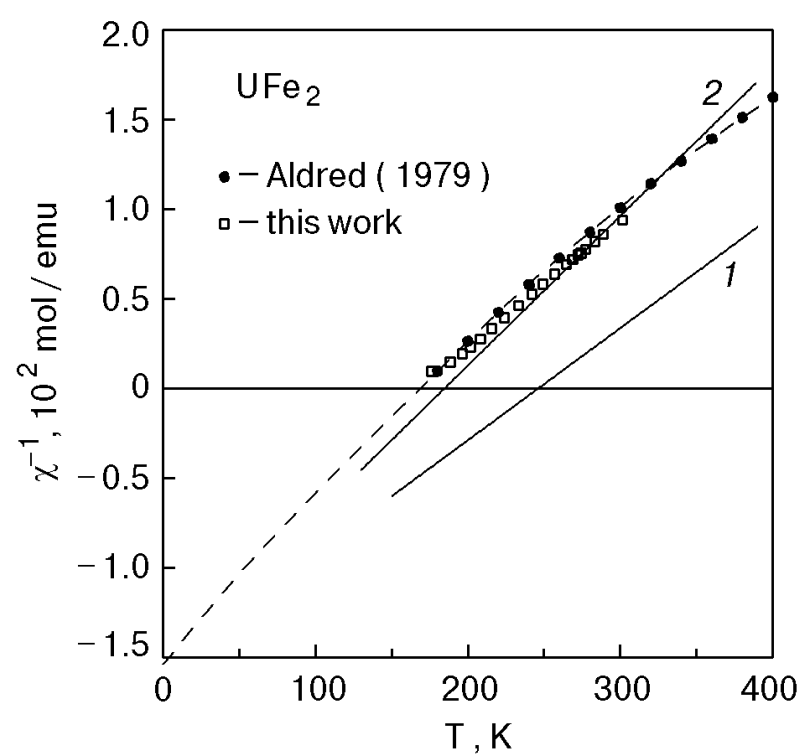

Fig. 6. Temperature dependence of the reciprocal magnetic susceptibility for $\mathrm{UFe}_{2}$. The model descriptions of Eq. (12) (curve 1) and Eq. (16) with $\lambda=0.2(\mathrm{~K} \cdot \mathrm{emu} / \mathrm{mol})^{-1}$ (curve 2).

ties of $\mathrm{UFe}_{2}$ based alloys appear to be important because of large exchange enhancement resulted from condition $\alpha \chi_{P} \sim 1$.

Following Fig. 5, the density of states curve for $\mathrm{UFe}_{2}$ at the vicinity of the Fermi level can be imagined as a peak $h$ in height and $\Delta$ in width which is placed on the background of height $N_{0}$ with $h / N_{0}<<1$ (see the insert in Fig. 5). The estimate $N_{0} \simeq 92$ (spin.Ry.cell $)^{-1}$ follows from the value $\chi_{P}^{0} \simeq 2.2 \cdot 10^{-4} \mathrm{emu} / \mathrm{mol}$, corresponding approximately to the peak pedestal. The value $h \simeq$ $\simeq 18(\text { spin } \cdot \text { Ry.cell })^{-1}$ results from estimate of the total density of state at the Fermi level for $\mathrm{UFe}_{2}$, $N \equiv N_{0}+h \simeq 110(\text { spin.Ry.cell })^{-1}$, obtained from Eq. (4) by extrapolation of the experimental $1 / \chi(T)$ dependence for $\mathrm{UFe}_{2}$ in the paramagnetic region to $T=0 \mathrm{~K}$ (see Fig. 6 , dashed line) that yields $\chi_{P}(0) \simeq 2.6 \cdot 10^{-4} \mathrm{emu} / \mathrm{mol}$. As evident from Fig. 5, the $\chi_{P}(n)$ peak for $T=250 \mathrm{~K}$ has $\delta n \simeq 1.5$ electrons per $3 d$ atom in width and contains of about

$S \sim \delta n\left(\chi_{P}^{\max }-\chi_{P}^{0}\right) /\left(\chi_{P}^{0}+\chi_{P}^{\max }\right) \simeq 0.15$ states $/$ cell.

With the $S$ and $h$ values mentioned above the estimate $\Delta \simeq 0.017$ Ry can be obtained if for simplicity the peak form is taken to be triangle. Thus, the set of the model parameters is

$$
\begin{gathered}
N_{0} \simeq 92(\text { spin } \cdot \text { Ry } \cdot \text { cell })^{-1}, \\
h \simeq 18(\operatorname{spin} \cdot \text { Ry } \cdot \text { cell })^{-1}, \quad \Delta \simeq 0.017 \mathrm{Ry}
\end{gathered}
$$


To check the validity of the model parameters estimated (11) we have applied them to describe the temperature dependence of the magnetic susceptibility for $\mathrm{UFe}_{2}$ by using Eqs. (2) and (3). With a linearized approximation for the Fermi-Dirac distribution function [25] the $\chi(T)$ is given by

$$
\frac{1}{\chi(T)} \simeq \frac{1}{\chi_{P}(0)}+\gamma T-\alpha
$$

with

$$
\gamma=\frac{5.55 h k_{B}}{4 \mu_{B}^{2}\left(N_{0}+h\right)^{2} \Delta}=\frac{5.55 k_{B}}{4 \mu_{B}^{2} S}\left(\frac{h}{N_{0}+h}\right)^{2}
$$

As a consequence, the temperature dependence of the magnetic susceptibility obeys the Curie-Weiss law (see Fig. 6) with parameters

$$
C=\gamma^{-1}, \quad \Theta=\frac{C}{\chi_{P}(0)}\left[\alpha \chi_{P}(0)-1\right] .
$$

The resulted estimates, $C \simeq 1.6 \mathrm{~K} \cdot \mathrm{emu} / \mathrm{mol}$ and $\Theta \simeq 250 \mathrm{~K}$, are in reasonable agreement with the experimental values $C=1.2-1.4 \mathrm{~K} \cdot \mathrm{emu} / \mathrm{mol}$ and $\Theta \simeq 170 \mathrm{~K}[3,12,17]$ if we keep in mind the simplified character of the $\chi(T)$ description.

The approach used gives only the general features of the electronic structure and its relation to the magnetic properties in $\mathrm{UFe}_{2}$. To get a more rigorous treatment of the experimental data some refinements of the analysis would be introduced. Thus, along with the electron scattering, the changes in the density of states for isoelectronic $\mathrm{UFe}_{2}$ based alloys may be due to deviation from the rigid band behavior caused by formation under alloying of the impurity subbands. In this case the DOS of alloy is represented as a superposition of the DOS for individual compounds in the ratio determined by their concentration. For $\mathrm{U}\left(\mathrm{Fe}_{1-x} \mathrm{~T}_{x}\right)_{2}$ alloys, a rough estimate of this effect gives $\Delta N / N \sim-0.07$ for $x=1$ provided that we neglect the scattering. A moderate $\Delta N / N$ value obtained suggests that DOS of $\mathrm{UMn}_{2}$ and $\mathrm{UCo}_{2}$ are not differ essentially from that of $\mathrm{UFe}_{2}$. So, the rigid band model is rather good approximation for these systems (see also [8]).

It should be noted that estimated value for the density of states at the Fermi level in $\mathrm{UFe}_{2}$, $N\left(E_{F}\right) \simeq 110(\text { spin.Ry.cell })^{-1}$, is found to be somewhat less then the calculated one, 143 (spin. Ry.cell) ${ }^{-1}$ $[7,9]$. To eliminate this discrepancy a more appropriate value of $\alpha$ in Eq. (4) should be chosen.

Furthermore, on closer examination of the magnetic susceptibility, we have to take into account for a substantial cancellation of the $5 f$ spin moments on the uranium atoms by the orbital contribution [9-11]. As a result, the magnitude of $N$ derived from the measured susceptibility appears to be underestimated.

In addition, the refined version of the Stoner model (4) is required as well. In particular, the spin-fluctuation mechanism (SF) could be taken into account. In the simplified form SF leads to an additional term in the molecular field parameter $\alpha$ $[26,27]$

$$
\alpha(T) \simeq \alpha(0)-\lambda T,
$$

where $\lambda$ is a constant determined by the band structure parameters [26]. Substitution of Eq. (15) into Eq. (12) gives

$$
\frac{1}{\chi(T)} \simeq \frac{1}{\chi_{P}(0)}-\alpha(0)+(\gamma+\lambda) T .
$$

As a result, the modified Curie-Weiss parameters are defined as

$$
C^{*}=\frac{1}{\gamma+\lambda}, \Theta^{*}=\frac{C^{*}}{\chi_{P}(0)}\left[\alpha(0) \chi_{P}(0)-1\right] .
$$

For $\mathrm{UFe}_{2}$, the upper limit $\lambda \simeq 0.8(\mathrm{~K} \cdot \mathrm{emu} / \mathrm{mol})^{-1}$ follows from the experimental value of the Curie constant provided the latter is completely determined by SF mechanism. Unfortunately, at present we have no direct quantitative estimate of the real role of $\mathrm{SF}$ in the magnetic properties in $\mathrm{UFe}_{2}$ and related alloys. It is notable that the better agreement between the model description (12) and experimental $\chi(T)$ dependence in $\mathrm{UFe}_{2}$ (Fig. 6) can be obtained by taking SF into account. In addition, because $C$ is approximately constant in $\mathrm{UFe}_{2}$-based alloys (see, for example, [12]), the SF parameter $\lambda$ can be assumed to be not critically dependent on concentration. From this fact combined with a small value $\lambda T$ in itself $[(\lambda T / \alpha) \ll 1$ for the temperature range under consideration] one may speculate that SF does not play an important role in the behavior of the magnetic susceptibility of $\mathrm{UFe}_{2}$ system under alloying.

\subsection{Pressure effects}

A phenomenological treatment of the experimental values of $d \ln \chi / d P$ can be given in the framework of the Curie-Weiss law (1) as 


$$
\begin{gathered}
\frac{d \ln \chi}{d P}=\frac{\chi_{0}}{\chi} \frac{d \ln \chi_{0}}{d P}+ \\
+\frac{\chi-\chi_{0}}{\chi}\left(\frac{d \ln C}{d P}+\frac{1}{T-\Theta} \frac{d \Theta}{d P}\right) .
\end{gathered}
$$

Since for $\mathrm{UFe}_{2}$-based alloys $\chi_{0} / \chi<<1$, a rough estimate $d \ln \chi_{0} / d P \approx d \ln \chi\left(\mathrm{UCO}_{2}\right) / d P \simeq-2 \mathrm{Mbar}^{-1}$ is assumed to be reasonable. Thus, the derivatives $d \ln C / d P$ and $d \Theta / d P$ can be evaluated from Eq. (18) by using the available experimental data for $d \ln \chi / d P$ at two different temperatures (Table). For $\mathrm{UFe}_{2}$ and $\mathrm{U}\left(\mathrm{Fe}_{1-x} \mathrm{Mn}_{x}\right)_{2}$ alloys with $x=0.1$, 0.25 and 0.3 , these values appear to be weakly dependent on the concentration and equal to

$$
\begin{gathered}
d \ln C / d P=-6 \pm 1 \mathrm{Mbar}^{-1}, \\
d \Theta / d P=-620 \mp 100 \mathrm{~K} \cdot \mathrm{Mbar}^{-1} .
\end{gathered}
$$

Notice that the estimate for $d \Theta / d P$ agrees closely with value $d T_{C} / d P \simeq-0.5 \mathrm{~K} / \mathrm{kbar}$ resulted for $\mathrm{UFe}_{2}$ [4, 15] and $\mathrm{U}\left(\mathrm{Fe}_{1-x} \mathrm{Mn}_{x}\right)_{2}$ [28] alloys. As to the pressure derivative of the Curie constant, its magnitude appears to be surprisely large. At least such value is not resulted from simple band approach (13), (14) which predicts $d \ln C / d P \sim 0$ provided the uniform deformation of the band is assumed. It seems likely that large pressure dependence of $C$ is a peculiar feature of $\mathrm{UFe}_{2}$-based alloys. The origin of this peculiarity is not clear.

In the context of the Stoner model, Eq. (4), the pressure effect, $d \ln \chi / d P$, is given by

$$
\begin{gathered}
\frac{d \ln \chi}{d P}=\frac{d \ln \chi_{P}}{d P}+\alpha \chi\left(\frac{d \ln \chi_{P}}{d P}+\frac{d \ln \alpha}{d P}\right) \equiv \\
\equiv \frac{d \ln N}{d P}+\alpha \chi\left(\frac{d \ln N}{d P}+\frac{d \ln I}{d P}\right) .
\end{gathered}
$$

It follows from Eq. (20) that the value of $d \ln \chi / d P$ for different temperatures and/or compositions of alloys can be approximated by a linear function of the magnetic susceptibility provided that the parameters $d \ln N / d P, d \ln I / d P$ and $\alpha(\propto I)$ are weakly dependent on temperature and composition. The experimental values of $d \ln \chi / d P$ for $\mathrm{U}\left(\mathrm{Fe}_{1-x} \mathrm{Mn}_{x}\right)_{2}$ and $\mathrm{U}\left(\mathrm{Fe}_{1-x} \mathrm{~T}_{x}\right)_{2}$ alloys are plotted against susceptibility in Fig. 7 . In accordance with Eq. (20), this dependence is close to a linear one, described by the following relation between the parameters:

$$
\frac{d \ln N}{d P}+\frac{d \ln I}{d P}=-0.16 \pm 0.03 \mathrm{Mbar}^{-1}
$$

$$
\frac{d \ln N}{d P} \approx-3 \mathrm{Mbar}^{-1}
$$

As seen from Eq. (21), a nearly full compensation for the pressure effects in the density of states $N$ and interaction parameter $I$ is found. In such a situation, the total effect $d \ln N / d P+d \ln I / d P$ appears to be strongly dependent on the details of the behavior of both components under alloying and with the temperature change. Probably, the latter is a reason for a stronger $d \ln \chi / d P_{\text {vs. }} \chi$ dependence at the origin of the coordinates (see inset in Fig. 7) which contains the room temperature data. The inclusion of the terms $(\gamma+\lambda) T$ of Eq. (16) into consideration leads to an additional contribution into the initial sum $d \ln N / d P+d \ln I / d P$ in Eq. (20):

$$
-\frac{(\gamma+\lambda) T}{\alpha}\left(\frac{d \ln (\gamma+\lambda)}{d P}+\frac{d \ln N}{d P}\right) .
$$

A rough evaluation of this term for $T=293 \mathrm{~K}$ can be made by using the experimental data for $\gamma+\lambda \simeq C^{-1} \simeq 0.8(\mathrm{~K} \cdot \mathrm{emu} / \mathrm{mol})^{-1}$ [see Eq. (17)], $d \ln (\gamma+\lambda) / d P \simeq-d \ln C / d P \sim 6 \mathrm{Mbar}^{-1} \quad$ and $d \ln N / d P \sim-3 \mathrm{Mbar}^{-1}$ which gives

$-\frac{(\gamma+\lambda) T}{\alpha}\left(\frac{d \ln (\gamma+\lambda)}{d P}+\frac{d \ln N}{d P}\right) \simeq-\begin{array}{r}0.15 \mathrm{Mbar}^{-1} \\ \text {. } 24)\end{array}$

With estimate obtained the resulted slope of $d \ln \chi / d P$ vs. $\chi$ dependence appears to be nearly twice as large as the initial one that is in a reasonable agreement with experimental data (inset in Fig. 7).

It should be noted that the corrections (24) do not depend obviously on a specific mechanism for

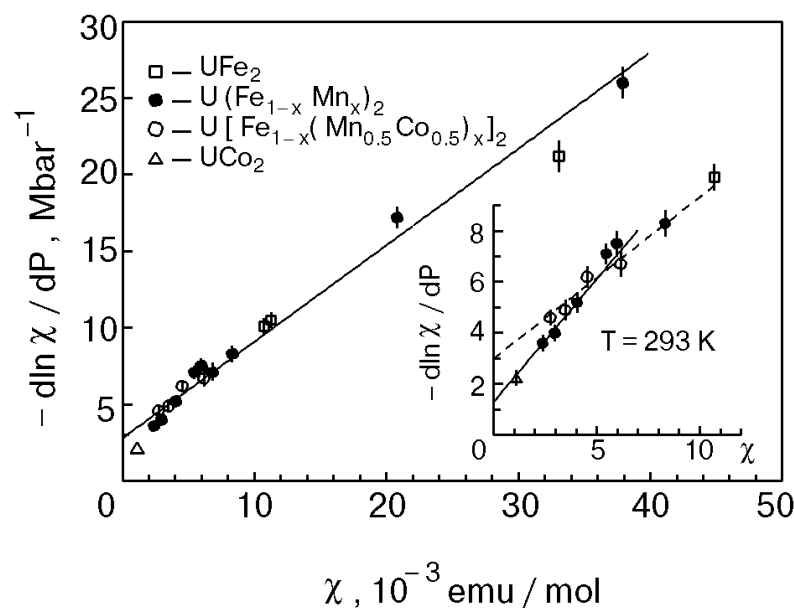

Fig. 7. Dependence of $d \ln \chi / d P$ on $\chi$ in $\mathrm{U}\left(\mathrm{Fe}_{1-x} \mathrm{Me}_{x}\right)_{2}$ alloys, $\mathrm{Me}=\mathrm{Mn},\left(\mathrm{Mn}_{0.5} \mathrm{Co}_{0.5}\right)$, Co. Inset: the data at $T=293 \mathrm{~K}$, the initial slope of the dependence is given by dashed line. 
the temperature dependence of the magnetic susceptibility and can be quantitatively included in an extended analysis of the pressure effects. In any case such inclusion remains the conclusion about a nearly complete compensation of pressure effects in $N$ and $I$ unchanged. Thus, on account of their small magnitude, the corrections mentioned above can be incorporated into the error bar for the total effect:

$$
d \ln N / d P+d \ln I / d P=-0.16 \pm 0.15 \mathrm{Mbar}^{-1} .
$$

Substitution of the bulk modulus value $B \simeq$ $\simeq 1.4 \mathrm{Mbar}$, obtained from the sound velocity study in a $\mathrm{UFe}_{2}$ single crystal [29], into Eq. (25) gives

$$
d \ln N / d \ln V+d \ln I / d \ln V=0.22 \pm 0.2 \text {. }
$$

It should be noted that $d \ln \chi / d P$ data in Fig. 7 correspond to alloys with different derivative of $\chi$ with respect to a number of conduction electrons per atom $n$ (see Fig. 4). Thus the lack of noticeable deviations of these data from the general linear run indicates that for $\mathrm{UFe}_{2}$ and its alloys effects of electron transfer under pressure are likely to be small. The conclusion obtained is confirmed by theoretical estimate for this effect [7].

In order to extract the change of $I$ with volume from Eq. (26), the value $d \ln N / d \ln V$ have to be known. As a first step, the estimate $d \ln N / d \ln V \simeq 2$ obtained from the experimental data in the inset of Fig. 7 can be appropriate, which agrees closely with value calculated for $\mathrm{UFe}_{2}$ in paramagnetic state [22]

$$
d \ln N / d \ln V=1.85 .
$$

The latter is assumed to be more realistic and has been used to obtain

$$
d \ln I / d \ln V=-1.6 \pm 0.2 .
$$

This evaluated derivative is close to that obtained for strong itinerant paramagnets-vanadium [20], palladium alloys [30], (Fe-Co)Si alloys [31], $\mathrm{Ni}_{3} \mathrm{Al}$ and $\mathrm{TiCo}$ compounds [32], for which the $d \ln I / d \ln V$ values fall in the range from -0.7 to -1.3 . On the other hand, these values differ essentially from the results of the LSDA calculations for $\mathrm{UFe}_{2}(-0.1[7,22])$ and for a number of $d$-metals and their compounds (from 0 to 0.2 [33]). Thus, it may be suggested that the LSDA method, employing the exchange-correlation potential of a uniform electron gas, is not well suited for an explanation of the volume dependence of $I$ in the systems with narrow bands such as $d$ - and $f$-metals. For these systems, the Hubbard approach, which takes into account the energy of the intraatomic
Coulomb repulsion $U$, is more appropriate description. In the context of this approach $[34,35]$ the relation between interaction parameter $I$ and the band width $W$ is given by

$$
\frac{d \ln I}{d \ln V}=\frac{d \ln W}{d \ln V} f(W / U, n) .
$$

Here, the coefficient $f \leq 1$ depending on the ratio $W / U$, the band filling $n$ and the crystal structure type. For $U=\infty, f$ appears to be unity and the band width becomes the only energy parameter yielding $|d \ln I / d \ln V| \leq|d \ln W / d \ln V| . \quad$ Typical estimates of $d \ln I / d \ln V$ for $d$-metals within the Hubbard model $[33,36]$ are in agreement with the experiment confirming the predominant role of short-range correlations in electron interaction for $d$-metals and their compounds, including $\mathrm{UFe}_{2}$.

\section{Summary}

The study performed makes it possible to conclude:

- Effects of deformation of the band under alloying caused by i) the electron scattering, ii) the assumed non-rigidity resulted from formation of the impurity subbands and iii) chemical pressure are comparable in their influence on the magnetic phase diagram with the band filling change.

- The Fermi level of $\mathrm{UFe}_{2}$ is found to be located just at the top of a small and narrow $N(E)$ peak. This peak is the only peculiarity in the density of states detected in the $\mathrm{U}(\mathrm{Mn}-\mathrm{Fe}-\mathrm{Co})_{2}$ system. The quantitative estimates for the peak parameters are obtained.

- The temperature and pressure dependence of the magnetic susceptibility in the $\mathrm{U}(\mathrm{Mn}-\mathrm{Fe}-\mathrm{Co})_{2}$ system is satisfactorily described by the enhanced Pauli spin contribution within the band model. Probably, the uranium spin and orbital contributions are roughly canceled at all these conditions considering their functional dependence on the band structure parameters to be the same.

- There is no noticeable pressure effect on the Fermi level caused by the relative shift of bands with different symmetry and the corresponding electron transfer between them.

- Nearly full compensation for the pressure effects in the density of states $N$ and interaction parameter $I$ is found.

- Value for volume derivative $d \ln I / d \ln V$ confirms the predominant role of the short-range correlations in the electron interaction in the compounds considered.

Some numerical discrepancy between the band model used and the experimental data for the mag- 
netic susceptibility can be attributed to neglecting of the orbital contribution as well as the spin fluctuations effect.

\section{Acknowledgments}

The present paper is closely connected with the name of academician B. I. Verkin, outstanding physicist and organizer of science. His pioneer works on the pressure effect on the band structure of metals have stimulated a search for methods to study weak magnetism under pressure as an effective tool for investigation of the electron states in alloys. B. I. Verkin not only took an active interest in these studies but he also directly contributed to the development of cooperation between the institutes represented by the authors of the paper. We consider it a great honor for us to devote gratefully our paper to the memory of B. I. Verkin.

The authors would like to thank Dr. O. Eriksson for supplying the detailed results of the band structure calculation [7] at different atomic volumes and Dr. A. B. Beznosov for valuable discussions. The experimental assistance of L. S. Litinskaya is gratefully acknowledged.

1. G. Hilscher, J. Magn. Magn. Mater. 27, 1 (1982); ibid. 25, 229 (1982).

2. G. H. Lander, A. T. Aldred, B. D. Dunlap, and G. K. Shenoy, Physica 86-88B, 152(1977).

3. A. T. Aldred, J. Magn. Magn. Mater. 10, 42 (1979).

4. J. J. M. Franse, P. H. Frings, F. R. de Boer, and A. Menovsky, Physics of Solids under High Pressure, J. S. Schilling and R. N. Shelton (eds.), North-Holland, Amsterdam, p. 181 (1981)

5. V. Sechovsky, Z. Smetana, G. Hilscher, E. Gratz, and H. Sassik, Physica 102B, 277 (1980).

6. A. M. Boring, R. C. Albers, G. H. Schadler, A. C. Lawson, P. Weinberger, and N. E. Christensen, Phys. Rev. B36, 5507 (1987).

7. O. Eriksson, B. Johansson, H. L. Skriver, and M. S. S. Brooks, Physica 144B, 32 (1986).

8. O. Eriksson, B. Johansson, M. S. S. Brooks, and H. L. Skriver, Phys. Rev. B40, 32 (1989) 9519.

9. M. S. S. Brooks, O. Eriksson, B. Johansson, J. J. M. Franse, and P. H. Frings, J. Phys. F18, L33 (1988).

10. O. Eriksson, M. S. S. Brooks and B. Johansson, Phys. Rev. B41, 9087 (1990).

11. B. Johansson, O. Eriksson, L. Nordström, L. Severin, and M. S. S. Brooks, Physica B172, 101 (1991).
12. R. Grössinger, G. Hilscher, J. Kamesberger, H. Sassik, G. Wiesinger, V. Sechovsky, and J. Toul, J. Magn. Magn. Mater. 29, 305 (1982).

13. G. R. Marpoe, Jr. and G. H. Lander, Solid State Commun. 26, 599 (1978).

14. A. I. Meskhishvili, V. A. Pletyushkin, V. K. Slovyanskikh, V. I. Chechernikov, and T. M. Shavishvili, Fiz. Metal. Metalloved. 45, 935 (1978)(in Russian).

15. P. H. Frings, J. J. M. Franse, and P. E. Brommer, $J$. Phys. C18, 1955 (1985).

16. J. Toul, V. Sechovsky, and G. Hilscher, Acta Phys. Slov. 31, 269 (1985).

17. E. Burzo and M. Văleanu, Rev. Roum. Phys. 29 (1984) 375 .

18. E. Burzo and M. Văleanu, J. Phys. F12, 3105 (1982).

19. A. S. Panfilov, Phys. Tech. High Pressure 2, 61 (1992) (in Russian).

20. A. S. Panfilov, Yu. Ya. Pushkar, and I. V. Svechkarev, Fiz. Nizk. Temp. 14, 532 (1988) [Sov. J. Low Temp. Phys. 14, 293 (1988)]

21. L. S. Litinskaya, A. S. Panfilov, A. Zentko, P. Diko, and J. Miskuf, Proc. Int. Conf. Physics of Transition Metals, Naukova Dumka Kiev, 1989 Pt. 1, 211.

22. Unpublished data of Ref. [7].

23. A. Hahn and W. Treutmann, Z. Angew. Phys. 26, 129 (1969).

24. V. N. Manchenko, A. S. Panfilov, and I. V. Svechkarev, Zh. Eksp. Teor. Fiz. 71, 2126 (1976) [Sov. Phys. JETP, 44, 1118 (1976)].

25. C. J. Kriessman and H. B. Callen, Phys. Rev. 94, 837 (1954).

26. M. Shimizu, Physica B159, 26 (1989).

27. T. Moriya and A. Kawabata, J. Phys. Soc. Jpn 34, 63 (1973); ibid. 35, 669 (1973).

28. L. R. Edwards and C. E. Olsen, Bull. Amer. Phys. Soc. 18 , 360 (1973).

29. T. P. Sorokina, G. M. Kvashnin, and A. M. Kapitonov, Fiz. Metal. Metalloved. (in Russian) 66, 1220 (1988).

30. A. S. Panfilov, Yu. Ya. Pushkar, and I. V. Svechkarev, $Z h$. Eksp. Teor. Fiz. 95, 751 (1989) [Sov. Phys. JETP, 68, 426 (1989)].

31. A. S. Panfilov, I. V. Svechkarev, and L. F. Romasheva, Fiz. Nizk. Temp. 19, 284 (1993) [Sov. J. Low Temp. Phys. 19, 200 (1993)].

32. P. E. Brommer, G. E. Grechnev, J. J. M. Franse, A. S. Panfilov, Yu. Ya. Pushkar, and I. V. Svechkarev, J. Phys.: Condens. Matter 7, 3173 (1995).

33. A. B. Kaiser, A. M. Oleś, and G. Stollhoff, Phys. Scr. 37, 935 (1988)

34. J. Kanamori, Prog. Theor. Phys. 30, 275 (1963).

35. N. D. Lang and H. Ehrenreich, Phys. Rev. 168, 605 (1968).

36. G. Stollhoff, A. M. Oles', and V. Heine, Phys. Rev. B41, 7028 (1990). 